\title{
The paretic pupil: its incidence and aetiology after keratoplasty for keratoconus
}

\author{
P. D. DAVIES AND M. RUBEN \\ Moorfields Eye Hospital, High Holborn, London, $W_{\mathrm{I}} V{ }_{7} A \mathcal{N}$
}

The occurrence of a paretic pupil as a complication peculiar to penetrating keratoplasty for keratoconus is said to have been first noted by a number of authorities, including Castroviejo and Paufique, but it was Urrets-Zavalia ( 1963 ) who first published his observations on a series of cases and suggested that the association of a fixed dilated pupil, iris atrophy, and secondary glaucoma constituted a specific syndrome which has since been frequently known by his name. He described five cases from 225 grafts in which the complete syndrome was present in two cases and a dilated pupil alone in three cases. Subsequently, Alberth and Schnitzler (1970) reported eight cases of paretic pupil, none with glaucoma, from a series of eighty penetrating keratoplasty operations. All these authors have related the onset of the syndrome to the postoperative use of mydriatics, particularly atropine, although others have felt that trauma plays a significant role. This paper attempts to establish in more detail the incidence, importance, and possible aetiology of the dilated pupil after keratoplasty.

\section{Cases}

We have examined 140 eyes from 108 patients with keratoconus who have undergone penetrating keratoplasty at the High Holborn branch of Moorfields Eye Hospital in the past 6 years. All these patients had what may be termed "advanced keratoconus", meaning that they had reached a stage where no visual improvement could be obtained with a contact lens and the preoperative keratometry gave a reading of less than 5.50 in at least one meridian.

\section{Operative technique}

A standard surgical procedure was followed in each case:

(i) Guttae pilocarpine 2 per cent. in three doses I $\mathrm{hr}$ preoperatively.

(ii) General anaesthesia.

(iii) A 7 or $8 \mathrm{~mm}$. penetrating graft was cut with a Address for reprints: P. D. Davies, F.R.C.S., Moorfields Eye Hospital,
High Holborn, London, WClV 7AN trephine and completed with scissors, a surgical microscope being used throughout.

(iv) A "watertight wound" was obtained and the anterior chamber re-formed with either normal saline or Miochol (I per cent. acetylcholine chloride) at the end of the operation. In the first 37 cases, between sixteen and 24 direct, interrupted, 8.0 monofilament nylon sutures were used to secure wound closure, and in the remaining 103 cases a continuous 10.0 monofilament nylon suture was used after the graft had been secured in position with four interrupted sutures of the same material.

(v) In 42 of the eyes operated upon, two or more basal peripheral iridectomies were performed immediately after the host corneal disc was excised, while in two other cases a small accidental iridectomy was noted to have been made. In the remaining 96 eyes no attempt was made to interfere with the iris.

(vi) NO MYDRIATIC was instilled postoperatively in any of the 140 eyes in this series.

\section{Results}

The 140 eyes examined in this study form a typical series of keratoconus cases, 93 per cent. attaining a corrected visual acuity of $6 / 12$ or better, and 87 per cent. a visual acuity of $6 / 9$ or better after keratoplasty (Table I).

Table I Visual results after keratoplasty: 140 eyes

\begin{tabular}{l}
$\begin{array}{l}\text { Corrected } \\
\text { visual acuity } \\
\text { No. of cases }\end{array}$ \\
\hline
\end{tabular}

PARETIC PUPIL

Eleven eyes with a fixed dilated pupil were noted ( 7.8 per cent.), but in four of these the paresis proved transient, and recovered spontaneously over a period 
of $\mathrm{I}$ to $\mathrm{I} 8$ weeks, completely in two cases and partially in the two other cases. This left seven eyes ( 5 per cent.) with permanently fixed and dilated pupils (Table II).

Table II Incidence of paretic pupil after keratoplasty: I 40 eyes

\begin{tabular}{|c|c|c|c|c|c|}
\hline \multirow{2}{*}{\multicolumn{2}{|c|}{$\begin{array}{l}\text { Post-operative } \\
\text { ( } 2 \text { wks after } \\
\text { keratoplasty) }\end{array}$}} & \multirow{2}{*}{\multicolumn{2}{|c|}{$\begin{array}{l}\text { Permanent } \\
\text { ( } 1 \text { yr after } \\
\text { keratoplasty) }\end{array}$}} & \multicolumn{2}{|c|}{ No. Recovered } \\
\hline & & & & \multirow[t]{2}{*}{ Partially } & \multirow[t]{2}{*}{ Completely } \\
\hline No. & Per cent. & No. & Per cent. & & \\
\hline I I & $7 \cdot 8$ & 7 & 5 & 2 & 2 \\
\hline
\end{tabular}

In only one of these eyes with a dilated pupil was evidence of glaucoma found, and in this eye, a flat anterior chamber had been present for 5 days after operation, before it re-formed spontaneously. Unfortunately this episode led to the development of extensive peripheral anterior synechiae and secondary glaucoma some 7 weeks later. In all the remaining I 39 eyes in this series, the anterior chamber was noted to be formed at the first dressing $24 \mathrm{hrs}$ after surgery. Our finding of glaucoma only in this one case complicated by a flat anterior chamber has similarities to the experience of Urrets-Zavalia ( 1963$)$; glaucoma occurred only in his two patients who had extensive peripheral anterior synechiae and one of these had a flat anterior chamber as a result of extensive iris prolapse through $180^{\circ}$ of the hostdonor corneal junction.

The fixed dilated pupil in each of the eleven cases mentioned above was present at the first dressing 24 hours after operation, or developed in the next 12 days; in particular, the four eyes with a transient paretic pupil were all originally noted to have fixed and dilated pupils at the first dressing. The presence of a paretic pupil does not seem to have affect adversely the outcome of the operation; all the eleven patients achieved a visual acuity of $6 / 12$ or better (Table III).

Table III Visual acuity of eyes with paretic pupils after keratoplasty

$\begin{array}{lllll}\text { Corrected visual acuity } & \frac{6 / 5}{\text { No. of cases }} & \frac{6 / 6}{5} & \frac{6 / 9}{4} & \frac{6 / 12}{1}\end{array}$

One of the interesting and previously unreported findings to emerge from this study was the presence of a partially dilated pupil in eighteen (19 per cent.) of the 94 cases which we were able to re-examine in the last 3 months. In this context, we would define a "partially dilated" pupil as one which is larger by at least $\mathrm{I} \cdot 5 \mathrm{~mm}$. than that of its unoperated fellow eye, and although both react to light and near fixation, the partially dilated pupil does so incompletely, remaining larger than its fellow for a given stimulus. These pupils do however constrict fully with topical miotics.

\section{IRIS ATROPHY}

Transillumination of the irides in the eyes comprising this study revealed a high incidence of iris atrophy. This was of two very different types:

(I) Focal atrophy (Figs I and 2)

The most common variety of iris atrophy (Table IV) found in eyes with both normal,

Table IV Incidence of focal iris atrophy after keratoplasty

\begin{tabular}{|c|c|c|c|c|}
\hline Pupil size & Paretic & Partial & Normal & All \\
\hline \multirow{3}{*}{$\begin{array}{l}\text { Number of cases } \\
\text { Focal atrophy No. } \\
\text { Per cent. }\end{array}$} & I I & 19 & 64 & 94 \\
\hline & 6 & 8 & 12 & 26 \\
\hline & 55 & 42 & 19 & 28 \\
\hline
\end{tabular}

partially dilated, and fixed dilated pupils after keratoplasty, consists of multiple focal areas in which the posterior pigmented epithelial layer of the iris is absent, the stroma however appearing normal to surface microscopy. The areas occur just beneath the host-donor corneal junction and are apparent immediately after surgery.

(2) Sector atrophy (Figs 3-6)

This very much less common variety of iris atrophy occurred in all seven eyes with a permanently dilated pupil and in one of the two eyes in which the pupil exhibited partial recovery after being initially fixed and dilated. Large areas of iris atrophy, involving all layers and extending up to the pupil margin, are characteristic with additional scattered areas of atrophy visible throughout the region of the sphincter pupillae. Although the dilated pupils in these cases were all noted within a few days of surgery, the atrophy did not develop for several months, the earliest being 2 months and the latest 5 months after operation.

\section{AETIOLOGY OF IRIS ATROPHY AND PARETIC PUPIL}

The more common focal iris atrophy must be traumatic in our opinion for two reasons:

(i) Its position relative to the host-donor corneal junction;

(ii) The fact that it can be detected immediately after surgery. 

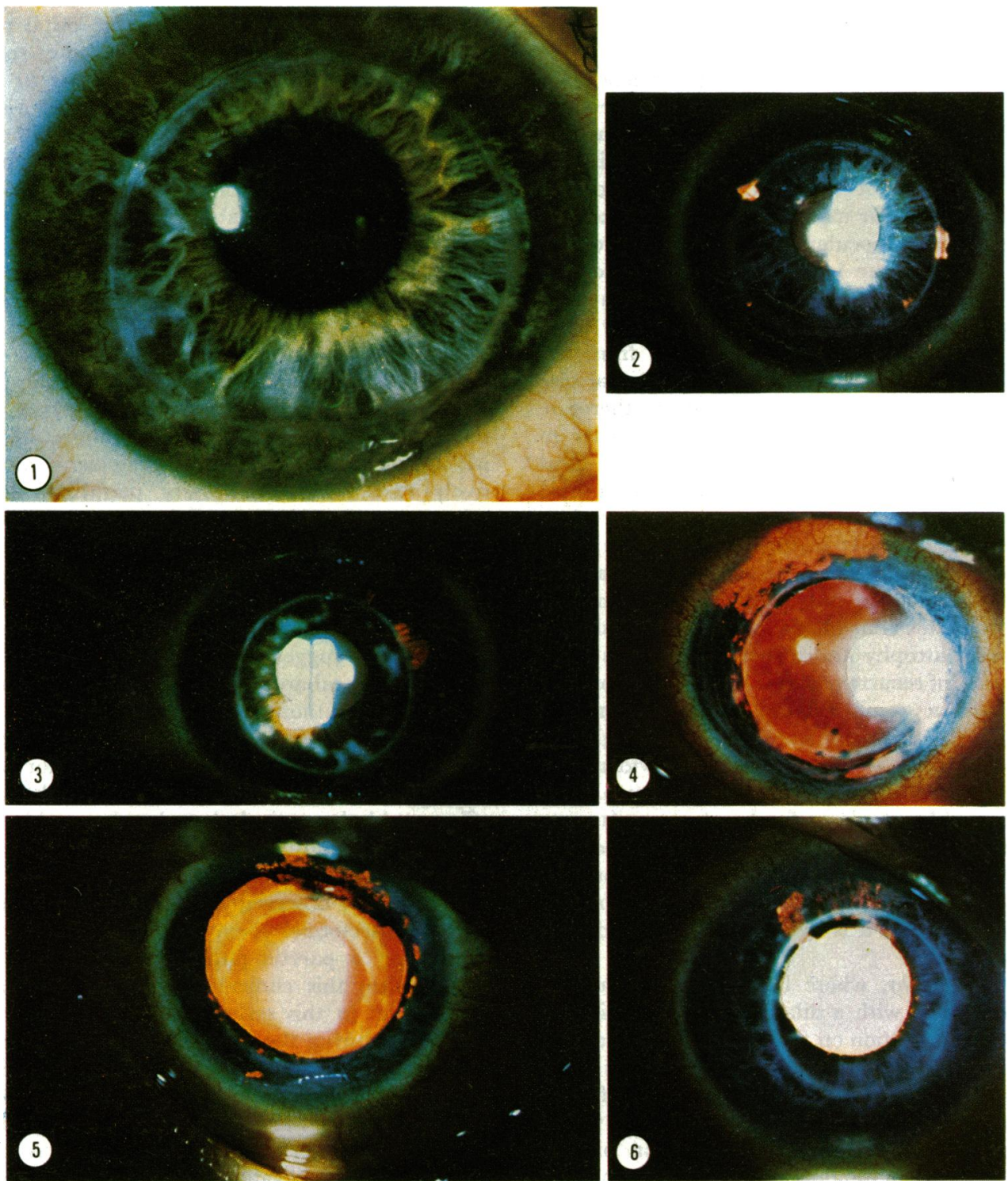

FIG. I Corneal graft of good clarity associated with normal pupil and visual acuity of 6/5. Surface microscopy of iris reveals no abnormality except a small iridotomy at io o'clock, produced accidentally by corneal scissors in completing excision of host corneal disc

FIG. 2 Focal iris atrophy. Retroillumination of iris in same eye as Fig. I reveals multiple areas, immediately beneath host-donor junction, where posterior pigment layer of iris is deficient. The similar appearance and position of these areas to that of the iridotomy suggests they have a common aetiology

FIG. 3 Focal iris atrophy. Another patient with a clear corneal graft, normal pupil, and visual acuity of 6/6, showing no abnormality of iris on surface microscopy but area of pigment loss on retroillumination

FIGS 4 AND 5 Sector iris atrophy. Retroillumination of iris in two patients with post-graft paretic pupils clearly showing large areas of atrophy involving all layers of iris. Scattered areas of atrophy also present throughout region of sphincter pupillae

FIG. 6 Sector iris atrophy. Less extensive area of atrophy in eye which exhibited partial recovery of fixed dilated pupil present in immediate postoperative period 
These factors further suggest that this is not a true atrophy but simply the result of trauma disturbing the pigment layer of the iris. It is inevitable, when completing the excision of the host-corneal disc, that the scissors may lightly touch the underlying iris and this seems to be the most likely cause of injury. Trauma of this nature is of course minimal and must moreover occur in all keratoplasty operations, although it is far more likely in cases of keratoconus where the intraocular contents tend to prolapse forwards, pressing the iris against the back of the cornea. This situation contrasts with that in nonkeratoconic eyes where the anterior chamber often remains formed, with the iris separated from the back of the cornea, even after the host-corneal disc has been excised. The ocular rigidity seems therefore to be abnormally low in keratoconus. In order to determine the general incidence of such focal trauma, we examined 127 eyes on which a corneal graft had been performed for reasons other than keratoconus, by the same surgeons using the same techniques over the same period of time as the keratoconus group. In only two ( $\mathrm{I} \cdot 5$ per cent.) of the eyes from this control group did we find similar areas of focal atrophy and in both these cases there was a history of recurrent pan-uveitis and secondary glaucoma as a result of underlying rheumatoid arthritis, a condition in which the iris might be expected to be abnormally fragile. The evidence is, therefore, that focal iris atrophy is very much more common after keratoplasty for keratoconus (19 per cent.) than after keratoplasty for other corneal pathology ( 1.5 per cent.), and furthermore its presence in eyes with both normal and paretic pupils indicates that it is not directly associated with the paretic pupil.

In sector iris atrophy, where large areas of atrophic iris are associated with a dilated pupil, it is difficult to explain the condition on the basis of direct trauma, because the atrophy develops some months after surgery and the onset of the dilated pupil. The failure of the pupil to constrict with Pilocarpine, which acts directly on the autonomic effector organs, indicates that traumatic denervation of the sphincter is not the cause of the dilated pupil. Therefore, if we also exclude direct trauma to the sphincter pupillae muscle as a cause of the mydriasis, then the paresis of this muscle would seem to be ischaemic in origin. Observation of the iris vessels during surgery reveals that they are dilated peripheral to the cut edge of the host cornea and relatively constricted axial to it (Fig. 7), an appearance which can be explained by a vascular strangulation phenomenon occurring as a result of the intraocular contents pushing the lens-iris diaphragm against the posterior surface and cut edge of the host cornea (Fig. 7). The consequent ischaemia paralyses the sphincter and the pupil dilates to a greater or lesser extent, the degree of ischaemic atrophy which subsequently develops in the muscle determining whether the pupil remains dilated or recovers. Alternatively, the sphincter could become ischaemic from occlusion of the greater arterial circle of the iris as a result of the iris being pressed against the back of the cornea by the intraocular contents, though such hypothesis does not explain the appearance of the iris vessels at operation that we have described above. Theoretically, fluorescein angiography performed during surgery should confirm whether an ischaemic strangulation phenomena does occur and indeed such a study was considered in this series but not proceeded with for ethical reasons.

A strangulation phenomena of this nature is put forward as the direct cause of the paretic pupil but does not explain why such pupils, like focal iris atrophy, are common in keratoconus and relatively rare after keratoplasty performed for any other corneal pathology. (We examined 246 nonkeratoconic eyes, matched for sex and age with the keratoconus group, and found only two cases of fixed dilated pupil, an incidence of 0.8 per cent. compared with 7.8 per cent. for the keratoconus group.) The suggestion is, therefore, that the iris is in some way abnormal in keratoconus, a hypothesis which some authorities feel is strengthened by the observation that even before surgery, the pupils of eyes with keratoconus remain dilated for a comparatively long time after mydriasis, although this effect could alternatively be related to a thin cornea allowing an unusually large quantity of drug to gain access to the anterior chamber.

It has been observed in the past that basal peripheral iridectomies seem to protect an eye from developing a paretic pupil after keratoplasty. We can confirm this clinical impression, since all the eleven eyes in this series in which a paretic pupil was noted during the immediate postoperative phase came from the group of 98 eyes in which no peripheral iridectomy was performed. No fixed dilated pupil was recorded in the 42 eyes on which a peripheral iridectomy was performed, a result which is statistically significant at a value of $P=0.05$. This apparent protection which the peripheral iridectomy affords the iris presumably functions by relieving the tendency to iris strangulation and pupil-block that we have described above.

Since we first began to suspect that iris strangulation might be significant in causing a dilated pupil after keratoplasty for keratoconus, we have modified our technique in an attempt to minimize this tendency. These modifications were:

(i) Preoperative Mannitol 20 per cent. $\times 250 \mathrm{ml}$. intravenous injection over $30 \mathrm{~min}$., from $1 \frac{1}{2}$ hrs before operation, in order to reduce vitreous volume. 

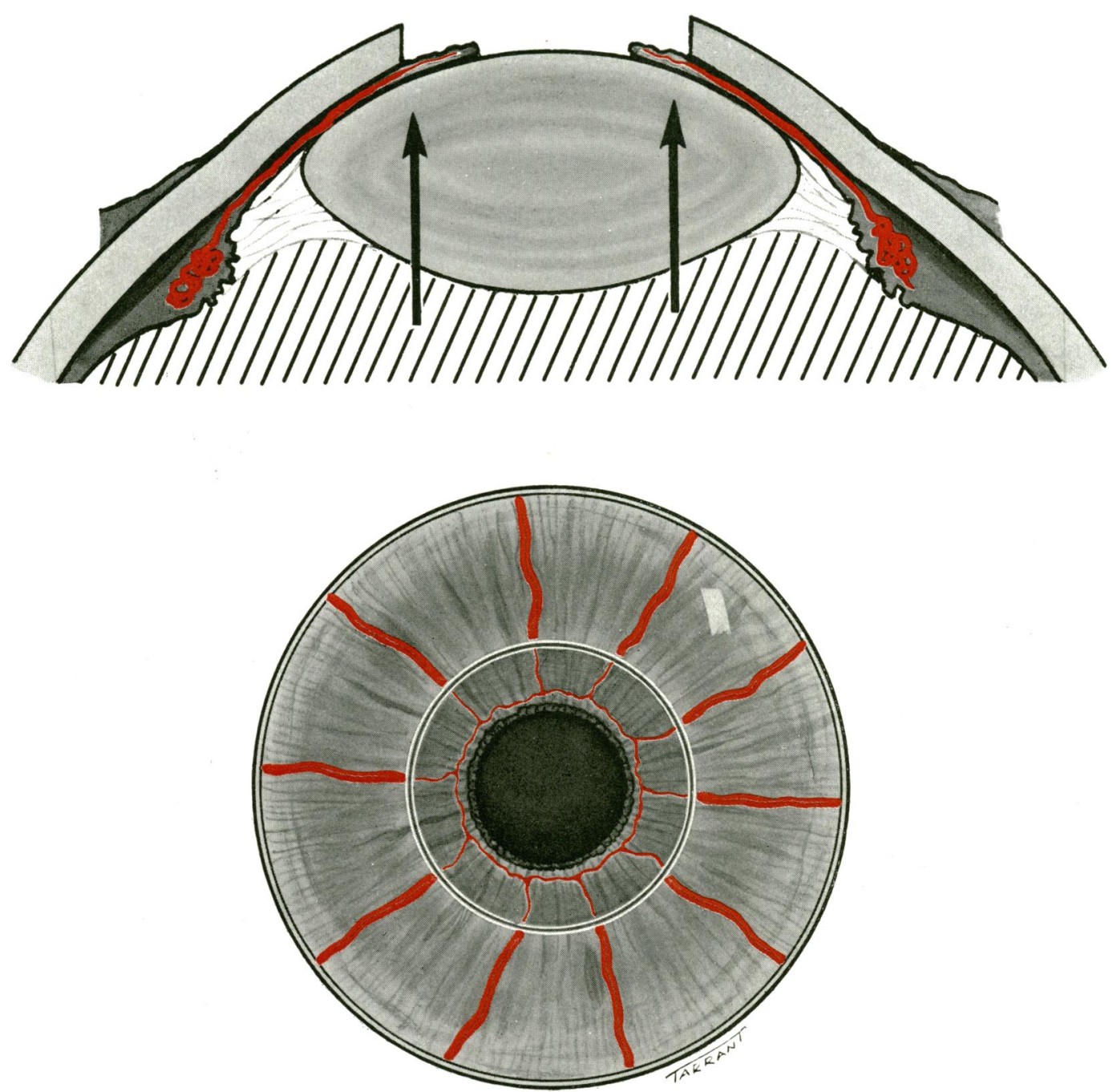

FIG. 7 Mechanism of relative iris strangulation. (a) Iris vessels may become occluded through compression against cut edge of host cornea as lens-iris diaphragm moves forwards

(b) Diagrammatic representation of how iris vasculature often appears through operating microscope after excision of host corneal disc

(ii) The use of muscle relaxants and intermittent positive pressure ventilation anaesthesia to reduce the intraocular pressure as much as possible.

(iii) Re-forming the anterior chamber as soon as possible during the operation and maintaining it while the continuous suture is completed.

We have so far performed 36 penetrating grafts for keratoconus using this technique, and there were only two paretic pupils, one of which did however partially recover over the next 3 months. These numbers are, of course, not yet statistically significant, but on the evidence of the relatively small number of cases it is obvious that these modifications in technique do not give absolute protection against the development of a fixed dilated pupil.

\section{Conclusions}

The present study reveals that pupillary abnormalities are common after keratoplasty for keratoconus and that, in addition to the fixed dilated pupils which we have found in 7.8 per cent. of eyes, varying degrees of partially dilated pupil frequently occur after 
operation. In our experience, glaucoma is not a sequel to the simple paretic pupil, a finding which confirms the results of the smaller series of Alberth and Schnitzler (1971); glaucoma thus seems to be no more a special complication of keratoplasty for keratoconus than it is of keratoplasty for any other corneal pathology. The paretic pupils can be explained on the basis of ischaemic atrophy of the sphincter pupillae muscle secondary to an iris strangulation phenomenon occurring during surgery in the manner we have discussed.

The relative frequency of a dilated pupil, together with the common finding of focal iris atrophy after minimal surgical trauma to the iris in cases of keratoconus, forces one to conclude that the pathology in this condition is not confined to the cornea but probably extends to the iris and possibly to the scleral envelope as well.

\section{References}

Alberth, B., and schnitzler, A. (1971) Klin. Mbl. Augenheilk., 159, 330

URRETS-Zavalia, A. (1963) Amer. $\mathcal{F}$. Ophthal., 56, 257 